Relations industrielles

Industrial Relations

\title{
Les changements technologiques: Une stratégie d'étude exploratoire
}

\section{Yves-Chantal Gagnon et Maurice Landry}

Volume 44, numéro 2, 1989

URI : https://id.erudit.org/iderudit/050500ar

DOI : https://doi.org/10.7202/050500ar

Aller au sommaire du numéro

Éditeur(s)

Département des relations industrielles de l'Université Laval

ISSN

0034-379X (imprimé)

1703-8138 (numérique)

Découvrir la revue

Citer cet article

Gagnon, Y.-C. \& Landry, M. (1989). Les changements technologiques: Une stratégie d'étude exploratoire. Relations industrielles / Industrial Relations, 44(2), 421-447. https://doi.org/10.7202/050500ar
Résumé de l'article

Cet article présente une stratégie d'investigation reliée à l'implantation de changements technologiques dans les milieux syndiqués.
Tous droits réservés @ C Département des relations industrielles de l'Université Laval, 1989
Ce document est protégé par la loi sur le droit d'auteur. L’utilisation des services d'Érudit (y compris la reproduction) est assujettie à sa politique d'utilisation que vous pouvez consulter en ligne.

https://apropos.erudit.org/fr/usagers/politique-dutilisation/ 


\title{
Les changements technologiques Une stratégie d'étude exploratoire
}

\author{
Yves-Chantal Gagnon \\ et
}

Maurice Landry

Cet article présente une stratégie d'investigation reliée à l'implantation de changements technologiques dans les milieux syndiqués.

Il est possible de distinguer deux catégories différentes d'études exploratoires. Il y a d'abord celles qui portent sur des sujets dont la pertinence ne semble pas faire problème mais qui, pour des raisons diverses, ont été peu ou pas explorés jusqu'ici. Le défi que posent ces études est celui de concevoir une stratégie d'investigation qui soit la plus appropriée possible compte tenu du fait que le terrain est riche en possibilités d'exploration mais pauvre en jalons pour les guider et que, par ailleurs, d'autres études suivront nécessairement.

La seconde catégorie d'études exploratoires se situe en amont de la première en ce qu'elles portent sur des thèmes dont la pertinence n'est pas pour l'heure suffisamment étayée pour justifier de s'y attaquer de plein pied et d'investir en conséquence, même si une argumentation sur leur intérêt potentiel peut être avancée. En somme, il s'agit dans ce cas de recherches dont le but est de savoir dans quelle mesure et jusqu'à quel point un sujet donné mérite d'être approfondi. Le défi de cette seconde catégorie d'études exploratoires consiste à concevoir une démarche permettant de confirmer ou d'infirmer cet intérêt potentiel et, dans l'affirmative, d'indiquer la direction que devraient prendre les études subséquentes. Idéalement, la stratégie d'investigation devrait alors être la moins «lourde» possible de sorte qu'en tout temps son coût d'utilisation reste en rapport avec sa pertinence espérée.

Une difficulté souvent rencontrée à l'usage de ces dernières stratégies vient du fait que lorsqu'elles donnent des résultats positifs en confirmant

* GAGNON, Y.-C., École nationale d'administration publique, Sainte-Foy, Québec.

LANDRY, M., Faculté des sciences de l'administration, Université Laval, Québec. 
l'intérêt de poursuivre plus avant, elles doivent du coup être abandonnées au profit d'autres stratégies mieux adaptées à la poursuite de la recherche et nécessitent de ce fait de nouvelles ressources et du temps additionnel. En d'autres termes, la difficulté vient alors du fait qu'une stratégie bien adaptée à l'investigation de la pertinence d'une problématique n'est pas nécessairement celle qui est la mieux adaptée à l'étude proprement dite de cette même problématique. Il y a alors discontinuité entre les deux ordres de stratégies. Ainsi, l'intérêt d'entreprendre l'étude d'un phénomène quelconque peut être établi en utilisant une méthode favorisant la mesure d'un certain nombre de paramètres jugés cruciaux alors que l'investigation de la nature et du comportement de ces mêmes paramètres fera très souvent appel à une toute autre stratégie.

Dans les disciplines d'orientation à la fois scientifique et professionnelle, cette discontinuité peut parfois être interprétée négativement parce que les recherches qui y sont faites ont souvent pour origine des préoccupations plus immédiates et suscitent des attentes de même nature. À notre avis, la difficulté issue de cette discontinuité n'est pas nécessairement incontournable. Le but de cet article est d'exposer et de discuter une stratégie d'investigation que nous avons utilisée pour nous attaquer à une problématique tombant dans la deuxième catégorie d'études exploratoires et reliée à l'implantation de changements technologiques dans des milieux syndiqués. Au départ, il s'agit donc d'établir plus solidement sa pertinence à partir d'un certain nombre d'indices pointant dans cette direction.

La stratégie d'étude exploratoire que nous présentons et illustrons ici à l'aide de ce cas, fait appel à des concepts tels ceux de «système d'action concret», de «pouvoir» et de «zones d'incertitude» qui sont au coeur de la théorie des organisations élaborée par Crozier et Friedberg (1977). Cette approche évite l'inconvénient de la discontinuité tout en permettant de bien indiquer les pistes de recherche intéressantes s'il s'en trouve. Elle permet de plus de ne pas exiger un investissement trop lourd en phase de démarrage de la recherche. Nous croyons enfin que son usage peut avantageusement être étendu ou adapté à plusieurs problématiques de recherche comme on en rencontre en relations du travail.

La première partie de l'article sert à exposer la problématique de départ et les raisons qui, a priori, nous ont fait croire à l'intérêt de l'explorer plus avant. Dans la deuxième partie, nous présentons les bases théoriques sur lesquelles nous appuyons notre démarche et montrons par la suite comment ces éléments théoriques ont été mis à contribution pour construire une carte préliminaire du terrain que nous voulions explorer. Nous utilisons cette carte préliminaire dans la troisième partie comme un instrument permettant à la fois de confirmer le bien-fondé de poursuivre plus avant l'étude et pour 
soulever en même temps un certain nombre de questions de recherche. Nous concluons enfin par quelques remarques en rapport avec l'usage de cette stratégie.

\section{LA PROBLÉMATIQUE DE DÉPART ET SON INTÉRÊT POTENTIEL}

Le succès suite à la mise en place des systèmes d'information organisationnels est une préoccupation centrale pour les concepteurs de systèmes en même temps qu'une source de grand intérêt et de fréquentes inquiétudes pour les gestionnaires (Robey et Farrow, 1982). La plupart des applications de la technologie de l'information présupposent, en effet, des changements significatifs prévisibles dans la façon dont le travail est fait et dans les qualifications de ceux qui sont directement affectés par ces changements (Hirschheim, 1985; Keen, 1985; Olson, 1982). L'implantation de nouvelles technologies, en plus d'impliquer un apport technique, contraint à revoir les procédés de travail, à redéfinir le contenu des postes de travail, à prévoir de la formation, etc. (Payeur, 1985). Plus encore, la technologie change souvent radicalement le visage de l'organisation, la nature du travail qui y est effectué, les rapports entre les acteurs, la dynamique de la compétition avec les autres entreprises et même la façon de faire des affaires. Il faut reconnaître, par ailleurs, que ces derniers changements sont beaucoup moins prévisibles parce que leur impact est à plus long terme (Miles, 1983; Chaumel, 1984). Traiter ces changements du seul point de vue technique accroît les chances d'échec: le nouveau système n'apporte pas alors tous les dividences escomptés ou encore son coût organisationnel devient excessif (Betcherman et McMullen, 1986; Desanctis et Courtney, 1983).

Ainsi, ce serait une erreur de négliger les aspects humains et organisationnels de ces changements car un système pleinement réussi sur le plan technique n'assure pas nécessairement son succès au plan organisationnel (Keen, 1976; White, 1984; Zmud, 1984). Dans ce contexte plusieurs auteurs ont souligné des succès de courte durée, des demi-succès ou des échecs avec les systèmes implantés (Bostrom et Heinen, 1977; Kling, 1980; Lucas, 1975; Roy, 1986). Lors d'une enquête informelle touchant 20 systèmes jugés réussis techniquement, 16 n'avaient pas atteint complètement les objectifs organisationnels qu'on leur avait fixés (Keen, 1981b). Les gestionnaires ont de façon croissante critiqué les professionnels en informatique pour leur relative incapacité à rencontrer pleinement leurs attentes face à ces nouveaux systèmes. Un nombre impressionnant de systèmes développés n'ont jamais été utilisés (Keen et Scott Morton, 1980). Au Québec, qu'il suffise de citer l'exemple du système Girafe 2000 du ministère de l'Éducation où un investissement de 7,8 millions a littéralement été perdu (Cliche, 1987). 
Pourtant, le futur prévisible semble devoir exiger encore plus des concepteurs de systèmes. Dans le contexte d'une concurrence provinciale, nationale et internationale de plus en plus serrée et accentuée par la déréglementation, l'élimination des quotas et la libéralisation des échanges commerciaux, où les coûts de la technologie ne cessent de décroître alors que ceux de la main-d'oeuvre augmentent (Keen, 1981a), les entreprises québécoises et canadiennes n'ont plus le choix. Il leur faut introduire de nouvelles technologies et raffiner leurs systèmes d'information pour améliorer sensiblement l'efficacité, la productivité et la rentabilité et ceci doit se faire rapidement face, notamment, à l'imminence d'un accord sur le libre échange avec les États-Unis. Tel est du moins l'avis de beaucoup d'experts (Betcherman et McMullen, 1986; Dussault, 1985; Fréchet, 1987; ministère d'État Sciences et Technologie, 1984).

Le tableau qui vient d'être dressé gagne encore en crédibilité quand on considère les chiffres suivants. Le Gouvernement du Québec a dépensé près de 500 millions de dollars pour l'informatique en 1985-1986 et prévoyait augmenter ce montant à 560 millions de dollars pour 1986-1987 (Cliche, 1987). Du côté canadien, une récente enquête auprès de 936 organismes montre que, dans $75 \%$ des cas, des changements technologiques significatifs ont été introduits entre 1980 et 1985 (Betcherman et McMullen, 1986). Aux États-Unis, en 1985, il y avait une station informatisée pour environ 20 travailleurs; la projection est d'une station par 2 ou 3 travailleurs d'ici 1990 (Keen, 1985). Au niveau mondial, l'industrie de la micro-électronique, avec ses 280 milliards de dollars en retombées économiques, a dépassé en importance l'industrie de l'automobile et on prévoit qu'elle deviendra la première industrie de la planète d'ici 15 ans, supplantant ainsi l'industrie pétrolière (Samson, 1985).

Bref, les enjeux soulevés par l'introduction des nouvelles technologies de l'information sont importants et les défis auxquels sont confrontés les concepteurs de systèmes sont de taille. Pour tenter de les relever, les concepteurs disposent de stratégies d'intervention nombreuses qui, malgré leur très grande variété, ont en commun de reconnaître l'importance, d'une part, de la collaboration de la haute direction et de ses représentants et, d'autre part, de la participation des usagers pour la réussite de l'implantation du système. La participation a pour but d'obtenir des usagers leur collaboration lors du développement puis à l'usage du système et de rendre ainsi ce dernier légitime à leurs yeux (Ferrand et Landry, 1987).

Dans la littérature en systèmes d'information organisationnels, «l'usager» occupe une place de choix. Cependant, il existe plusieurs catégories «d'usagers» et, jusqu'à maintenant, ce sont principalement ceux pour qui le système est construit qui ont reçu le plus d'attention. Étrangement, le 
groupe de ceux qui produisent les intrants ou reçoivent les extrants bruts du système, les usagers-opérateurs, qui sont indéniablement très directement concernés par le développement de nouveaux systèmes et qui, par surcroît, ont la caractéristique unique d'être souvent regroupés en syndicat et d'avoir ainsi le «comportement de groupe» le plus accentué, n'ont pas vraiment été étudiés. On a manifesté tout au plus un souci pour leur entraînement au nouveau système du genre ... «tu fais ceci», «tu ne touches pas à cette clélà» et, un intérêt pour l'impact que les changements informatiques décidés en d'autres lieux pouvaient avoir sur la qualité de leur vie au travail.

Nous n'avons pu recenser aucune recherche qui s'intéresse spécifiquement aux usagers-opérateurs et à l'influence de leur groupe formel, le syndicat, sur l'attitude qu'ils adoptent à l'occasion de l'implantation d'un système et sur l'impact que cette attitude peut avoir sur le succès du système. Peut-être est-ce parce que leur influence est jugée négligeable! De prime abord cependant, d'autres points de vue sont tout aussi plausibles et soutenables. C'est ainsi qu'en paraphrasant Crozier et Friedberg (1977), on pourrait également avancer que l'usager-opérateur n'est pas seulement une main, comme tend à le traiter le taylorisme et à sa suite une bonne part de la littérature en systèmes d'information, ni non plus seulement un coeur comme le souhaiterait Argyris (1971), mais également une tête qui agit en fonction de ses intérêts perçus et au mieux de ses connaissances. Qu'en est-il au juste de leur influence? Et si leur attitude s'avérait déterminante pour l'acceptation du système?

Les constats et interrogations qui précèdent justifient amplement à nos yeux d'entreprendre une étude exploratoire dans le but de confirmer ou infirmer l'intérêt de poursuivre plus avant dans cette direction. En effet, l'importance potentielle des questions qui sont soulevées ici dépasse le simple cas des systèmes d'information organisationnels. Derrière celles-ci, c'est une dimension importante du problème plus général de l'étude des conditions de réussite de ce qu'il est convenu d'appeler le virage technologique qui est soulevée.

\section{FONDEMENTS THÉORIQUES DE LA DÉMARCHE ET CARTE PRÉLIMINAIRE DU TERRAIN À EXPLORER}

Comme nous sommes confrontés à un terrain relativement inexploré en systèmes d'information, il nous est apparu utile de dresser d'abord une carte préliminaire de ce terrain pour mieux nous orienter par la suite. Par ailleurs, cette carte ne saurait être dressée au hasard. Elle doit être construite en s'appuyant sur des fondements théoriques solides. C'est pourquoi, avant de dresser la carte proprement dite, il importe d'exposer d'abord la théorie sur laquelle elle prend appui. 
Nature et origine de la notion de système d'action concret

La carte du terrain, appelée ici "système d'action concret», tente d'identifier, de caractériser puis de relier entre eux les principaux acteurs impliqués dans l'activité d'implantation de systèmes. Un système d'action concret se différencie d'un organigramme en ce que ce dernier décrit les relations formelles qui lient entre eux l'ensemble des dépositaires des différents postes dont est composée une organisation. L'organigramme reflète la pérennité des relations d'autorité entre ces divers postes. Le système d'action concret tente plutôt de représenter la relative stabilité des relations de pouvoir entre les divers acteurs, parties prenantes à un ensemble de problématiques données. Ces relations de pouvoir peuvent varier d'une problématique à l'autre parce que, pour chacune d'elles, les acteurs impliqués ainsi que les zones d'incertitude pertinentes qui sont à l'origine du pouvoir varient. Globalement cependant, il est possible d'observer un certain équilibre dans ces relations.

Crozier et Friedberg (1977), à qui nous empruntons le concept de système d'action concret pour l'adapter à notre problématique, postulent que dans les systèmes sociaux, il n'y a pas de champ neutre. Les structures organisationnelles ne peuvent être assimilées à de simples courroies de transmission d'un niveau hiérarchique à l'autre, de sorte que tout y soit entièrement réglé ou contrôlé a priori. L'organisation ne contraint jamais complètement les acteurs qui y oeuvrent. Ceux-ci gardent continuellement une marge de liberté qui leur vient des ressources dont ils disposent (savoir, ressources matérielles, poste hiérarchique, etc.) et de la relative imprévisibilité de leur comportement face aux autres qui leur est conféré par le degré de discrétion qu'ils peuvent exercer dans l'usage de ces ressources. Parce qu'ils contrôlent des zones d'incertitude, ils possèdent une marge de liberté et il en découle un pouvoir correspondant. Chaque acteur dispose donc sur les autres acteurs d'un certain pouvoir qui est à la mesure de la pertinence de la source d'incertitude qu'il contrôle face à eux. Le comportement de cet acteur doit par conséquent s'analyser comme l'expression d'une stratégie rationnelle visant à utiliser son pouvoir au mieux pour accroître ses «gains» à travers sa participation dans l'organisation.

C'est dans ce contexte que Crozier et Friedberg introduisent le concept de «jeux» et qu'ils le relient à celui de «système d'action concret» défini comme suit: «[...] un ensemble humain structuré qui coordonne les actions des participants par des mécanismes de jeux relativement stables et qui maintient sa structure, c'est-à-dire la stabilité de ses jeux et les rapports entre ceux-ci, par des mécanismes de régulation qui constituent d'autres jeux» (p. 246). Un acteur, pour pouvoir disposer d'une source de pouvoir face aux 
autres, est obligé de satisfaire partiellement leurs attentes à son égard. En ce sens, ceux-ci deviennent une contrainte pour lui. Il doit accepter certaines règles qui assurent le maintien de sa relation avec eux et permettent à chacun de continuer à jouer et à enregistrer des gains personnels. Ces règles viendront limiter sa liberté et encadrer ses négociations avec les autres membres de l'organisation. Le jeu est donc un mécanisme concret grâce auquel les hommes structurent leurs relations de pouvoir et les régularisent tout en laissant à chacun une marge de liberté. Il devient donc l'instrument à la base du construit d'action collective qui assure la coopération nécessaire entre acteurs sans supprimer leurs possibilités de poursuivre des objectifs contradictoires. Le construit d'action collective contraint à la fois les acteurs dans leurs choix tout en dépendant de ces choix pour son maintien.

Dans cette optique, le fonctionnement d'une organisation est vu comme le produit de l'adaptation d'un ensemble d'individus ou groupes par une série de jeux auxquels ils acceptent de participer. Les règles formelles ou informelles établies, en définissant les possibilités de gains et de pertes des uns et des autres, délimitent un éventail de stratégies rationnelles qu'ils peuvent adapter s'ils veulent que leur engagement dans l'organisation serve leurs buts personnels ou, du moins, ne les contrarie pas. Le système d'action concret doit permettre de mettre en évidence ces jeux.

\section{Son adaptation dans le cadre de notre étude}

La mise en évidence du système d'action concret dans lequel s'insère une problématique donnée constitue une stratégie d'investigation tout à fait intéressante parce qu'elle permet de mieux comprendre les enjeux qui structurent les relations entre les groupes d'acteurs impliqués. Mais cette stratégie est lourde. Elle ne peut se faire ni dans l'abstrait ni à partir d'une rationalité $a$ priori. Pour pouvoir dresser de tels systèmes d'action concrets, il faut recourir à une observation directe et approfondie des attitudes, des comportements et des stratégies des acteurs par l'évaluation de leurs ressources respectives et des contraintes de toutes sortes qui limitent leur liberté et influencent leurs actions. Cette recherche-action n'est pensable que si on dispose d'une période prolongée d'observation, de moyens financiers importants et d'un terrain qui s'y prête. C'est pourquoi, elle nous apparaissait mal convenir, telle qu'elle, à une étude exploratoire comme la nôtre: il nous fallait l'adapter. Or, nous disposions de deux sources distinctes d'information, la littérature en systèmes d'information organisationnels et celle en relations du travail. Chacune d'elles, bien que non suffisante pour les fins qui nous intéressent ici, complétait l'autre dans une certaine mesure. C'est ainsi que nous avons pu élaborer, à partir de ces deux sources d'informa- 
tion, de notre expérience du domaine et des déductions qu'il nous apparaissait logique de faire, une esquisse préliminaire du système d'action concret exigeant un investissement beaucoup plus léger mais nous permettant quand même de mieux comprendre le rôle des usagers-opérateurs et de saisir leur importance à l'occasion de l'implantation de systèmes.

Même si elle permet des économies appréciables de temps et d'argent, il est bien évident que la stratégie de substitution que nous utilisons ici ne peut prétendre à toute la richesse d'information qu'autorise une stratégie classique de recherche-action. C'est la raison pour laquelle nous ne parlons ici que «d'esquisse préliminaire», ce qui est d'ailleurs suffisant pour une étude exploratoire comme la nôtre. Il importe de rappeler également que cette limitation est partiellement compensée par le fait que l'esquisse préliminaire peut, par la suite, être soumise à un processus de validation par étapes qui convient également bien pour le type d'étude que nous poursuivons ici. Ainsi conçue, l'élaboration de l'esquisse préliminaire du système d'action concret sert à établir la pertinence et le potentiel de certaines pistes d'investigation qui pourront par la suite être scrutées plus en profondeur.

\section{L'esquisse préliminaire du système d'action concret dans lequel s'insère l'action des usagers-opérateurs}

Afin de ne pas alourdir indûment le texte, nous ne présentons ici que d'une façon schématique l'esquisse préliminaire du système d'action concret dans lequel les usagers-opérateurs sont impliqués. Le graphique 1 identifie les principaux acteurs concernés par ce système d'action concret, indique en termes généraux leur rôle et spécifie enfin les relations qui les unissent à l'occasion de l'activité d'implantation de systèmes. Ce graphique sera brièvement commenté ici après quoi nous tracerons un portrait plus détaillé de deux groupes d'acteurs qui se sont révélés être particulièrement importants en cours d'investigation.

Dans le graphique 1, les usagers-décideurs désignent ceux qui contrôlent complètement ou partiellement les ressources utilisées dans le système et qui ont le pouvoir d'influencer le développement du système comme c'est le cas pour la haute direction d'une organisation (Ciborra et Maggiolini, 1981). Les usagers-gestionnaires sont ceux qui supervisent au nom des usagers-décideurs le processus de conception ou l'opération du système et qui travaillent en collaboration avec le concepteur. Théoriquement, ils servent de lien entre les usagers-décideurs et les concepteurs mais leur connaissance du terrain fait en sorte que leur rôle peut facilement dépasser celui de simples agents de liaison. Ils peuvent être en fait les véritables artisans des politiques informatiques de l'entreprise que ne font alors qu'entériner par la 
suite les usagers-décideurs. C'est à leur niveau que se négocie la convention collective avec le syndicat local et que s'interprètent les clauses pertinentes relatives aux changements technologiques. Les clients du système sont ceux que le système aide directement dans l'accomplissement de certaines de leurs tâches. Ce sont, par exemple, les usagers des guichets automatiques on encore les gestionnaires pour qui un système expert a été construit (Mumford, Land et Hawgood, 1978). Le rôle des usagers-opérateurs est directement associé au système devenu opérationnel. Comme nous l'avons déjà mentionné, ce sont eux qui produisent les intrants ou encore reçoivent des extrants bruts du système qui a été pensé en fonction des clients. Les usagersopérateurs sont indispensables pour le fonctionnement journalier du système mais ils n'ont pas de pouvoir direct pour le changer (Hirschheim, 1985; Zmud, 1979a et b). Le système qu'utilisent les clients est en quelque sorte le fruit du travail d'interprétation des besoins des clients par le concepteur ainsi que de l'activité journalière des usagers-opérateurs. Les termes de la convention collective devraient donc normalement influencer les rapports entre le concepteur et les usagers-opérateurs.

Le concepteur est celui qui développe le système en collaboration avec les usagers-gestionnaires et les clients. Il est, sinon le responsable unique, du moins le maître d'oeuvre habituel de l'opérationalisation du système. Le concepteur est l'expert incontesté pour tout ce qui concerne l'aspect technique du développement. À ce titre, il contrôle une source importante d'incertitude face aux autres acteurs. Par exemple, son poids est souvent déterminant dans les choix technologiques qui doivent être faits et qui peuvent s'avérer plus ou moins contraignants sur le plan humain, social et organisationnel. De plus, la contrainte imposée par ces choix technologiques peut être amplifiée par le moment où ils s'exercent. Plus ceux-ci sont faits tôt dans le processus de développement, plus fortes tendent à être ces contraintes (Clegg et Kemp, 1986; Sandberg, 1985). C'est ainsi que le processus de développement peut être perçu ou non comme dominé par des contraintes technologiques et l'action du concepteur n'est pas neutre à cet égard.

C'est dans ce contexte que prend toute son importance l'affirmation à l'effet que les dimensions humaines et sociales semblent traitées comme de simples contraintes pour les concepteurs. Traditionnellement, ceux-ci ont vu leur rôle comme étant d'abord de développer et d'implanter des systèmes techniquement à point, même lorsque ces systèmes sont utilisés par des nontechniciens (Miles, 1983). Clegg et Kemp (1986) ont estimé qu'encore aujourd'hui près de $90 \%$ des efforts en temps et en argent sur les projets de changements technologiques sont consacrés aux questions reliées à la technique. Ainsi, on retrouve des projets majeurs en bureautique où la fièvre technologique conduit à escamoter la planification de l'implantation, laquelle devient un véritable exercice de chirurgie technologique (Miles, 1983; Cliche, 1987; Morin, 1986). 


\section{Graphique 1}

Esquisse préliminaire du système d'action concret

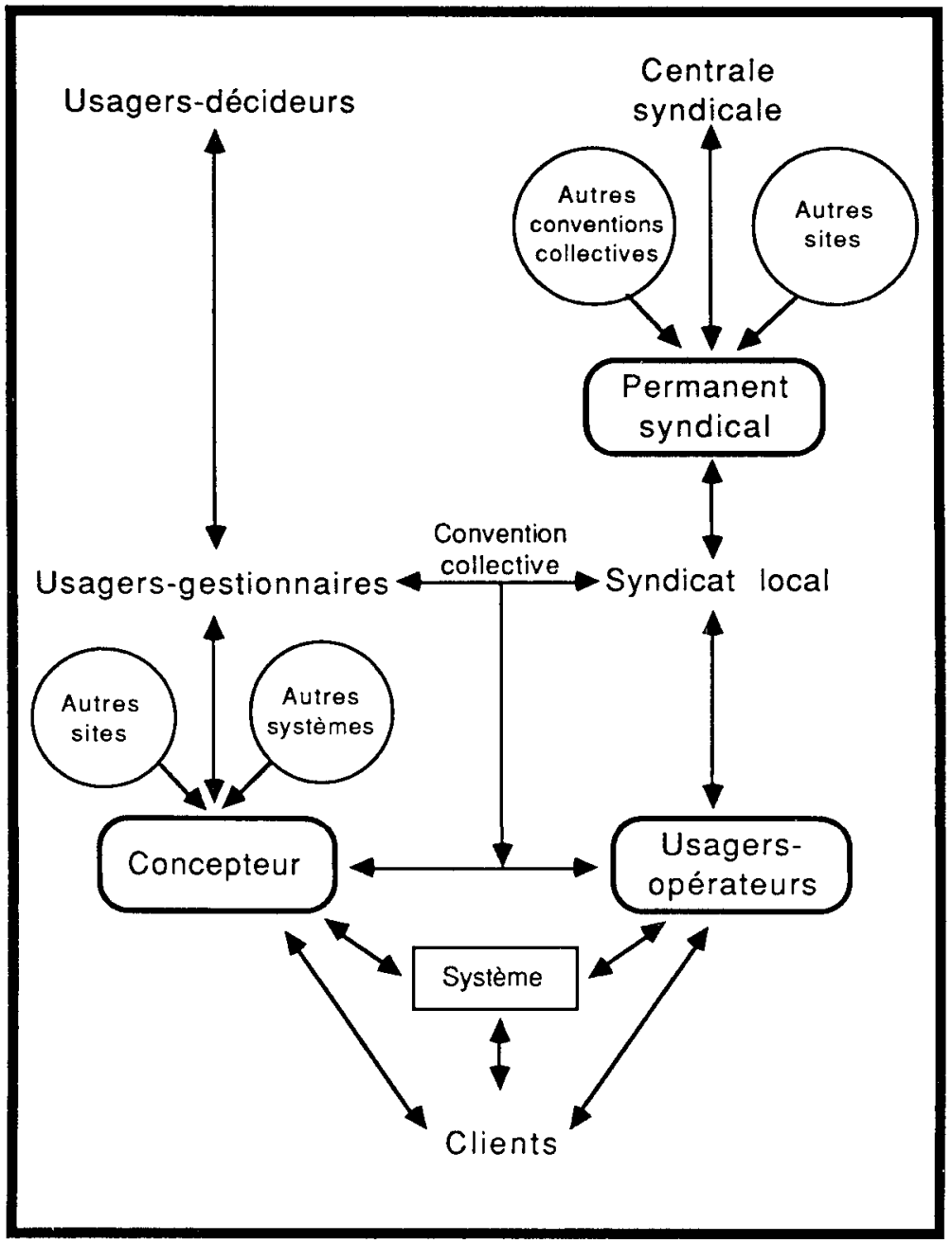


Par ailleurs, la prise en considération de la dimension sociale de l'implantation requiert un nécessaire appel à la coordination, à la communication et aux échanges soutenus entre l'informaticien et les différentes catégories d'usagers. Or, les traits de personnalité du concepteur ainsi que sa formation sont des éléments pouvant expliquer le peu d'importance qu'il semble réserver aux aspects humains et sociaux de la conception de systèmes. Il y a une différence substantielle entre la personnalité et la culture des informaticiens qui construisent les systèmes et les usagers qui les utilisent (Keen, 1981a; McKenney et Keen, 1974). Pour certains, cela explique la traditionnelle coupure entre concepteurs et opérateurs (Lafargue, 1986). L'informaticien-type préfère travailler seul et a tendance à réduire ses relations interpersonnelles au minimum (Mercier, 1986). Ainsi, dans une recherche auprès de 1229 informaticiens dans une centaine de compagnies en Australie et en Grande-Bretagne, il est apparu que les deux tiers étaient introvertis, alors que l'on retrouvait un ratio inverse dans la population en général. De plus, quatre-vingts pour cent d'entre eux étaient du type «penseur» dans l'échelle du «Myers-Briggs Type», c'est-à-dire qu'ils tendaient à prendre des décisions sur une base logique et impersonnelle (Berardo, 1983; Lyons, 1985). Qui plus est, la formation du concepteur privilégie clairement l'aspect technique et quand il accède à ce poste par promotion interne, c'est souvent après avoir été successivement programmeur et analyste, fonctions encore plus techniques (Clegg et Kemp, 1986; Lyons, 1985). Tout cela concorde avec la remarque de Hirschheim (1985) à l'effet que la formation des concepteurs, en sciences humaines, est souvent déficiente.

Enfin, la tâche du concepteur est telle que ce dernier semble devoir être constamment incité à privilégier l'atteinte de résultats à court terme. Un projet n'attend pas l'autre et ceux-ci ne concernent pas toujours la même unité organisationnelle. Souvent les concepteurs proposent ou acceptent des échéanciers impossibles à rencontrer. Leurs estimations ne couvrent souvent que l'analyse, le développement et le codage proprement dit et sont souvent faussées par un facteur de 2 à 10 (Keen, 1985). À plus long terme, c'est sur un site différent de celui sur lequel il travaille présentement qu'on a le plus de chances de retrouver le concepteur. Si tout ne marche pas comme prévu, c'est à d'autres qu'incombera alors la tâche de faire les modifications envisageables. Par ailleurs, si le concepteur peut relativement bien prévoir les conséquences à court terme de l'implantation de systèmes, sa connaissance devient beaucoup plus limitée pour les effets à moyen et à long termes. C'est donc à court terme que le concepteur est le plus en mesure de prédire les effets des changements qu'il implante et de rassurer les usagers en conséquence.

Il semble irréaliste de vouloir comprendre le rôle des usagers-opérateurs sans s'attarder à leur groupe d'appartenance, le syndicat. En effet, 
dans le domaine des relations du travail on s'intéresse de très près au changement technologique qu'on définit comme tout changement effectué dans la façon dont une entreprise gère, conçoit et fabrique les produits ou services qu'elle offre (Vallée, 1986). Cela inclut les changements organisationnels qu'entraîne l'implantation de systèmes et, du coup, justifie l'apparition de l'organisation syndicale locale et de sa centrale syndicale à l'occasion de l'implantation d'un nouveau système (Sandberg, 1985).

On ne s'entend pas sur les conséquences que peuvent avoir sur les travailleurs, surtout à long terme, les changements provoqués par les nouvelles technologies. Ainsi certaines études, principalement du côté syndical, font état de pertes d'emplois, de déqualification, d'augmentation de la charge de travail et du contrôle (Allaire, 1985; Bureau international du travail, 1986; Dommergues, 1984; Lamoureux, 1983; Payeur, 1983; Ropp, 1987; Tremblay et al., 1983). D'autres, au contraire, parlent de gains d'emplois (les pertes dans certains secteurs étant compensées par des gains plus grands dans le secteur de la technologie), de hausse de qualification, d'une plus grande autonomie dans le travail et d'un enrichissement des tâches (Attewell et Rule, 1984; Franz et al., 1986; Olson et Turner, 1985). L'obtention de ces résultats contradictoires résulte sans doute en partie de la perspective d'analyse adoptée. Mais elle est aussi due à divers autres facteurs, comme le degré d'automatisation, les types d'application, les secteurs d'implantation et la structure organisationnelle (Fréchet et al., 1987). L'influence de ces facteurs est d'ailleurs conséquente avec le fait énoncé plus haut à l'effet que l'implantation de systèmes doit aussi être vue comme un changement organisationnel et implique donc bien d'autres aspects que la seule introduction du système proprement dit.

Il est évident que le syndicat ne peut se désintéresser des conséquences sociales de l'implantation de systèmes. De fait, à l'inverse du concepteur, le syndicat est souvent perçu comme un acteur d'abord social pour qui la technique est une contrainte. S'il s'intéresse à la composante technique, c'est pour mieux voir comment elle pourrait bénéficier socialement à ses membres (Sandberg, 1985). Par ailleurs, les conséquences sociales des changements technologiques se manifestent à la fois à court et à long termes. Ce qui nous amène à faire une distinction entre les préoccupations premières des centrales syndicales et celles des syndicats locaux.

À cet égard, il est utile de noter la différence dans l'horizon d'intérêts entre le syndical local et sa centrale syndicale. Le syndicat local se situe directement à l'interface entre, d'une part les usagers-opérateurs et leurs préoccupations et, d'autre part, les gestionnaires de l'entreprise. Il a avant tout comme objectif de défendre les intérêts immédiats de ses membres, 
alors que la centrale syndicale se chargera plus spécifiquement de la stratégie plus globale et à plus long terme. Par rapport aux changements technologiques, ces différences jouent également.

Jusqu'à ce jour, selon plusieurs, les employés syndiqués auraient souvent été frappés négativement par les changements technologiques (Bureau international du travail, 1986; Gagnon, 1985; Poppel, 1983). Ce sont des changements devant lesquels ils ont souvent été placés sans communication ou information préalable (Dussault, 1985). Face à cette perception négative, le syndicat, surtout au plan local, doit fournir à ses membres des résultats immédiats et concrets de ses actions. Il doit à court terme tenter d'éviter, ou à tout le moins de diminuer, les conséquences sociales négatives des changements technologiques touchant directement les travailleurs (Sandberg, 1985). Pour ce faire, il dispose d'une arme de taille: un contrôle plus ou moins grand sur la collaboration de ses membres au processus de changement. Au niveau des centrales syndicales, des préoccupations plus globales à moyen et long termes font jour (Sandberg, 1985). En effet, pour bien comprendre les impacts possibles de l'introduction de nouveaux systèmes, il faut considérer plusieurs facteurs comme la propriété de l'information, la confidentialité, la sécurité, la relation de pouvoir, le changement des buts et des relations (Miles, 1983; Keen, 1981a). Dans ce contexte, il se peut que sur un site donné, il n'y ait pas d'effets négatifs à court terme occasionnés par les changements technologiques, mais que des effets négatifs, à plus long terme se soient fait sentir dans d'autres sites. L'un des rôles des permanents syndicaux de la centrale, affectés à plusieurs sites, est d'ailleurs de s'assurer que de telles situations soient évitées.

De quelles ressources disposent les permanents syndicaux? D'une part, ils constituent le lien privilégié par lequel les ressources de la centrale sont utilisées pour appuyer les actions du syndicat local. D'autre part, ils possèdent une expérience des changements technologiques et de leurs effets sur d'autres sites lors de ses transactions avec le syndicat local et avec la direction de l'entreprise. En fait, on peut croire qu'ils disposent face au syndicat local d'un pouvoir d'expertise équivalent à celui que possède le concepteur vis-à-vis les usagers-gestionnaires.

En général, on peut affirmer que l'implantation de systèmes implique des changements organisationnels plus ou moins importants. Tout changement s'accompagne d'une incertitude réelle ou perçue pour les individus et les groupes qui sont touchés (Caruth et al., 1985; Chaumel, 1984; Hirschheim, 1985), et, dans ce sens, il comporte un risque. Il y a des coûts et des bénéfices au changement pour l'organisation, les groupes et chaque acteur organisationnel (Keen, 1985; New et Singer, 1983). Ces coûts et bénéfices peuvent être financiers, comme la variation du salaire, mais aussi non 
financiers, tels un changement de statut, la variation de la charge de travail, l'apprentissage d'un nouveau système, la modification des relations d'amitié, de pouvoir, de prestige etc... Les individus ou les groupes, à la suite d'un changement, peuvent voir leur sort ou bien s'améliorer ou encore se détériorer (Filiatrault, non daté).

L'esquisse préliminaire du système d'action concret que nous venons de dresser suggère que le développement d'un système n'est pas un processus neutre qui prend place dans un champ non structuré; au contraire, il est l'occasion et le lieu de conflits potentiels entre des intérêts, des aspirations et des valeurs qui mettent en jeu autre chose que les seules technologies à partir desquelles le développement est accompli (Salomon, 1982). Il est donc permis de se demander si l'accrochage est inévitable. En effet, il se dégage, des écrits recensés plus haut, que le concepteur de systèmes est principalement préoccupé par la dimension technique et les effets à court terme de l'implantation de systèmes, qu'il contrôle une incertitude clef, le savoir d'expertise, et qu'il répond d'abord à la direction de l'entreprise. La centrale syndicale, pour sa part, se concentre principalement sur l'aspect social, vit beaucoup d'incertitude par rapport aux effets à moyen et à long termes du changement technologique et contrôle par ailleurs certaines sources d'incertitude. Le syndicat local enfin, se préoccupe principalement de l'impact à court terme de l'implantation de nouvelles technologies et n'est pas également dépourvu de ressources utilisables à l'occasion du changement.

\section{UN DÉBUT DE CONFIRMATION EMPIRIQUE DE L'ESQUISSE PRÉLIMINAIRE DU SYSTÈME D'ACTION CONCRET}

Dans l'optique d'une étude exploratoire, l'esquisse préliminaire d'un système d'action concret n'est que la première étape servant à établir la pertinence et le potentiel de certaines pistes d'investigation. En effet, comme cette esquisse préliminaire n'est dressée qu'à partir de la littérature, un début de confirmation empirique peut s'avérer une mesure additionnelle appropriée. C'est ce que nous avons fait ici en sélectionnant un groupe d'acteurs qui, selon l'esquisse préliminaire, apparaissait comme particulièrement important et qui, de plus, était relativement accessible.

\section{Caractéristiques de l'échantillon}

Nous rendrons compte ici des résultats empiriques obtenus à la suite des entrevues faites auprès de permanents des centrales syndicales québécoises, intimement impliqués dans la problématique qui nous intéresse ici. 
Nous avons en effet rencontré dix personnes, neuf hommes et une femme, qui occupaient des fonctions permanentes dans les grandes centrales syndicales québécoises (CSN, CEQ, FTQ, CSD) et qui avaient une expérience significative dans des situations de négociation à l'occasion de changements technologiques. Compte tenu du type de recherche poursuivie, ce nombre nous est apparu suffisant, d'autant plus que la population totale se situe autour de deux cents. Ces répondants ont été sélectionnés, selon leur disponibilité, au hasard parmi une liste de permanents qui avaient plusieurs années d'expérience au cours desquelles ils avaient eu à intervenir à plusieurs reprises, à titre de conseiller d'un syndicat local, dans des situations d'implantation de systèmes d'information au sein d'organisations québécoises. Cette liste avait été préalablement établie lors d'une rencontre avec un employé du service de la recherche de chaque centrale syndicale.

Les entrevues se sont échelonnées sur deux semaines et ont duré en moyenne une heure cinquante minutes chacune. Lors de ces rencontres, nous procédions toujours initialement à une mise en contexte, afin de créer un climat de confiance. Nous vérifiions également si le permanent répondait pleinement à nos critères de sélection. Par la suite, nous abordions de façon plus spécifique les expériences pertinentes du répondant pour ensuite cheminer à travers les questions de notre schéma d'entrevue qui étaient essentiellement basées sur ce qu'avait dégagé l'esquisse préliminaire du système d'action concret mis à jour.

Parmi ces répondants, un seul avait suivi des cours en informatique lors de sa formation universitaire (langage de programmation «Fortran»). L'ensemble de ces permanents syndicaux cumulaient quatre-vingt-dix-sept années d'expérience dans le milieu syndical, soit une moyenne de près de dix années chacun. Tous ces répondants ont eu dans leurs fonctions, à un titre ou à un autre, à conseiller plus ou moins régulièrement des syndicats locaux, regroupant tous des usagers-opérateurs, notamment à l'occasion de l'introduction de changements technologiques dans leur milieu. Ces répondants ont tous eu à travailler sur l'implantation de systèmes tels des systèmes bureautiques, des systèmes transactionnels, des sytèmes d'aide à la décision, de la robotique ou des outils de production informatisés.

Les secteurs plus spécifiquement et directement couverts par les répondants syndicaux étaient l'éducation (soutien et enseignement), les assurances, le textile, l'alimentation, la confection de vêtements, la construction, le gouvernement fédéral et les sociétés d'État, les municipalités, les affaires sociales, les chantiers maritimes et les pâtes et papier. La taille des entreprises touchées par l'action des répondants varie de la PME, c'est-à-dire de cinq employés à la grande multinationale comptant plusieurs milliers d'employés. 


\section{Les résultats des entrevues}

\section{La pauvreté des relations entre le concepteur et les usagers-opérateurs}

D'une façon générale, notre démarche empirique tend à confirmer l'esquisse préliminaire du système d'action concret qui vient d'être dressé. C'est dire que cette esquisse, à quelques exceptions près dont nous reparlerons plus loin, représente raisonnablement bien le système d'action concret tel que le voient les permanents syndicaux avec qui nous avons eu des entrevues. Une première exception concerne la relation qui y est présumée entre les concepteurs et les usagers-opérateurs. L'esquisse fait voir une relation directe et réciproque entre ces deux groupes, relation qui devait être influencée par les dispositions touchant les changements technologiques contenues dans les conventions collectives. Au-delà des exigences de la convention collective, l'existence de cette relation pouvait également s'expliquer par le fait que la littérature en systèmes d'information organisationnels (SIO) insiste beaucoup sur l'importance de tenir compte des usagers en général et de les faire participer pleinement à l'implantation des systèmes.

Or, il ressort de nos entrevues qu'il y a rarement une communication directe entre les concepteurs et les usagers-opérateurs. Lorsqu'elle se produit, elle est à sens unique allant du concepteur aux usagers-opérateurs et vise uniquement soit à transmettre des directives d'utilisation du système, soit encore à recueillir certaines informations spécifiques sur le travail accompli par les usagers-opérateurs. En ce qui concerne l'implantation de systèmes, c'est avec les gestionnaires, principalement ceux qui sont chargés des ressources humaines, que se font tous les contacts avec les usagers-opérateurs via leurs représentants syndicaux locaux et les permanents syndicaux des centrales qui interviennent au niveau local. À toutes fins utiles, le lien de communication entre le concepteur et les usagers-opérateurs est pour le moins ténu, superficiel et à sens unique. Il est très important d'être conscient de l'existence de cette perception d'un manque de communication concrète entre les concepteurs et les usagers-opérateurs ou leurs représentants syndicaux. En effet, ce manque d'échange est source de nombreuses impressions et perceptions négatives, réelles ou pas, qui ont une énorme influence sur le comportement des usagers-opérateurs et de leurs représentants. On peut difficilement espérer modifier ce comportement aussi longtemps que dans l'esprit des usagers-opérateurs et de leurs représentants persistera l'actuel système d'action concret.

Soulignons également le lien plus intime que prévu qui existe entre les permanents syndicaux et les syndicats locaux. Nous ne pouvons affirmer que les deux groupes forment un tout car, comme prévu, leurs horizons de 
préoccupation sont différents. Cependant, leurs actions sont nettement et clairement à maints égards imbriquées. Dans certains cas, le permanent agit comme porte-parole du syndicat local auprès des gestionnaires de l'entreprise tant pour la négociation que l'application de la convention collective. Ceci semble encore plus marquant quand il s'agit de traiter d'implantation de changements technologiques.

\section{Les usagers-opérateurs: des acteurs importants}

Les résultats de notre démarche nous amènent aussi à confirmer l'importance du groupe des usagers-opérateurs pour la réussite de l'implantation des systèmes d'information. Ce groupe, répétons-le, a été négligé dans la littérature en SIO où on ne s'est pas particulièrement préoccupé jusqu'ici de la variable syndicale ni de l'étude des phénomènes de groupes. Cet oubli trouve peut-être sa source dans le fait que pris individuellement le poids des usagers-opérateurs peut sembler négligeable; cependant, le système d'action concret dans lequel s'insère leur action a permis de mettre en relief leur grande importance via le rôle de leur syndicat. Les usagers-opérateurs et leur groupement syndical interviennent presque toujours à un moment ou à un autre à l'occasion de l'implantation d'un système. Ils interviennent même à la limite pour neutraliser les effets espérés par le concepteur à la suite de la mise en place du nouveau système, lorsqu'ils estiment ne pas pouvoir se faire entendre autrement. L'un des répondants donne comme exemple une institution d'enseignement où les employés continuent à maintenir, contrairement aux directives de leur employeur, un système manuel parallèle sept ans après l'introduction d'un nouveau système informatisé. D'autres permanents rencontrés citeront des cas où les systèmes ont dû être retirés et un cas où la faillite d'une PME serait attribuable en grande partie à l'informatisation ratée des opérations. Ils interviennent enfin au niveau de la législation et des conventions collectives qui ont une influence sur l'implantation de systèmes d'information. Ces interventions, le plus souvent à tendance défensive, visent à forcer le déplacement des règles du jeu à leur avantage.

Une relation foncièrement mauvaise entre les concepteurs et les usagers-opérateurs ou leur syndicat

Au plan des perceptions exprimées par les permanents syndicaux à propos des concepteurs de systèmes, il y a de mauvaises notes. Nos répondants croient unanimement que les concepteurs sont orientés à $99,9 \%$ vers la technique et cela parce que leur formation est avant tout technique. Les concepteurs sont poussés par la vente de leurs systèmes au patron. Cette vente se 
fait d'ailleurs uniquement sur la base d'une productivité accrue à très court terme. Toujours selon les permanents syndicaux, les concepteurs sont continuellement mis au défi en ce sens par le patron qui leur confie leurs mandats.

Pour les répondants, les concepteurs ne semblent pas prêter beaucoup d'intelligence aux usagers-opérateurs. Ces derniers se sentent perçus par les concepteurs comme des gens qui ont peur du progrès, comme des quantités négligeables et comme des personnes qu'il faut organiser sans qu'elles ne puissent positivement y contribuer elles-mêmes. La considération de leur point de vue retarderait le travail du concepteur. Les répondants croient également que les concepteurs perçoivent les syndicats très négativement, comme des «paquets de problèmes» et des organismes qui s'opposent au progrès.

À une exception près, les permanents rencontrés ont souligné que les concepteurs intimident les usagers-opérateurs. Pour l'un d'eux, par exemple, les travailleurs posent beaucoup de questions aux concepteurs et ils n'ont jamais de réponse satisfaisante. Même lorsqu'ils proviennent de l'intérieur de l'organisation, les concepteurs sont considérés comme des étrangers, des gens de l'extérieur. Un autre permanent souligne que les concepteurs ne parlent pas le même langage que les travailleurs et prétendent tout connaître; c'est pourquoi les travailleurs ne les aiment pas et les craignent. Quant au répondant qui fait exception, il affirme qu'on ne peut avoir peur des concepteurs puisqu'on ne les voit jamais. Concernant l'aspect technique du travail du concepteur, il faut souligner que nos répondants sont très conscients qu'ils n'ont pas l'expertise nécessaire pour discuter avec les concepteurs. Ils doivent limiter leur intervention à certaines dimensions de l'aspect social qu'ils connaissent, comme la protection des travailleurs, la santé et la sécurité, les clauses de conventions collectives. L'un des permanents souligne de plus que, contrairement à l'employeur, ils n'ont pas les ressources financières nécessaires pour embaucher des experts-conseils dans ce domaine et ils n'en sont pas eux-mêmes.

Sans doute qu'un minimum de contacts entre les deux groupes permettraient à tout le moins de modifier quelque peu de telles perceptions. Mais de l'avis de tous les permanents, jamais les représentants syndicaux n'ont de contact avec les concepteurs, qu'ils soient internes ou externes à l'organisation. En ce qui a trait aux usagers-opérateurs, on souligne également que très rarement les concepteurs les rencontrent pour voir le travail qu'ils font. Quand cela se fait, il n'y a pas de véritable communication puisque l'usageropérateur ne sait pas la plupart du temps pourquoi le concepteur le questionne. On mentionne enfin qu'un secret constant entoure le travail du concepteur. 
Tout ceci montre bien que la relation entre les concepteurs et les syndicats, telle que présentement perçue par les permanents syndicaux, rend difficile la réussite de l'implantation de systèmes d'information lorsque les usagers-opérateurs sont syndiqués. Avec une telle perception, les syndicats ont tendance à «opposer» plutôt qu'à intégrer la dimension sociale à la dimension technique propre à la conception de systèmes.

\section{Les effets des changements technologiques sont difficilement prévisibles et influencent peu le concepteur dans les choix de développement}

Les personnes rencontrées reconnaissent qu'on ne peut prévoir toutes les conséquences qu'entraînera l'implantation de systèmes, d'autant plus qu'il y a souvent des effets pervers qui surgissent à la suite de ces implantations. Il y a trop de variables intervenantes sur lesquelles personne n'a de contrôle et qui dépendent de chaque entreprise, de chaque secteur, de chaque région, de chaque employé touché par cette implantation. L'un des répondants fait remarquer, par exemple, que demain nous serons probablement dans le libre-échange ou encore qu'il pourrait y avoir une autre crise du pétrole. D'autre part, on souligne que les implantations se font souvent secteur par secteur, sans tenir compte que c'est de façon systémique que l'entreprise se transforme. Les permanents mentionnent aussi que les effets d'un nouveau système peuvent être très différents à court et à long termes. Ainsi un système peut avoir des effets négatifs pour les travailleurs à court terme (diminution d'emplois) alors qu'à long terme ces effets peuvent devenir positifs (augmentation du nombre d'emplois) et vice versa. À court terme, la technologie n'est sans doute qu'un outil, alors qu'à long terme, elle peut révolutionner.

D'ailleurs, selon les permanents syndicaux, les concepteurs ne se soucient pas des effets que peuvent avoir les systèmes développés sur les employés. Ils sont uniquement préoccupés par l'aspect productivité à court terme de leur système. Dans l'enseignement, par exemple, on souligne qu'avant qu'on les y force, les concepteurs n'avaient aucune préoccupation des effets pédagogiques et sociaux de leurs systèmes. Par ailleurs, au plan de l'échéancier d'implantation, on estime qu'il serait possible d'établir des calendriers plus réalistes tout en atteignant mieux les buts poursuivis.

En ce qui a trait aux effets possibles non pris en compte par les concepteurs, les répondants soulignent aussi qu'ils auraient besoin d'être rassurés, non seulement en ce qui touche les travailleurs directement visés, mais aussi les autres employés de l'organisation. Ceci notamment pour leur permettre d'influencer l'attitude que les travailleurs développeront par rapport à l'acceptation de ces changements. Pour être rassurés, les membres des syndicats 
auraient besoin de comprendre les changements et ce qu'ils peuvent avoir comme conséquences. Malheureusement, l'absence de communication amplifie chez eux l'effet d'incertitude.

L'ensemble des répondants sont tout à fait convaincus que les concepteurs pourraient concevoir des systèmes pour atteindre les mêmes buts tout en touchant différemment les travailleurs. Il n'y a donc pas de déterminisme technologique selon eux. On donne souvent comme exemple l'ergonomie et la santé et sécurité au travail (bruit des appareils électroniques, vapeur d'acide, etc.) en soulignant que ces appareils pourraient exécuter les mêmes fonctions tout en protégeant mieux la santé des travailleurs. Souvent les concepteurs agissent comme s'il y avait un déterminisme technologique et invoquent qu'on ne peut donner suite aux demandes syndicales pour cause d'impossibilité technologique. Ceci, même si souvent c'est le patron qui transmet cette réponse provenant du concepteur.

En conclusion, on peut avancer que si les effets des changements technologiques sont non prévisibles, les syndicats savent qu'ils ne peuvent être complètement rassurés quant à ces effets, puisqu'on ne les connaît pas. Par ailleurs, les syndicats sont suspicieux sur la façon dont l'imprévu sera traité lorsqu'il se présentera. D'autre part, les permanents syndicaux sont convaincus que les concepteurs pourraient atteindre les mêmes buts tout en touchant moins négativement les travailleurs; cependant, ils ne savent pas «comment» y parvenir. Finalement, les syndicats se sentent souvent confrontés par les concepteurs à une argumentation basée sur un déterminisme technologique, alors qu'ils n'y croient pas et ne sont pas armés pour la réfuter.

\section{Les concepteurs travaillent à très court terme et semblent accorder peu de temps à l'implantation qui est habituellement mal ou pas planifiée}

L'ensemble des répondants soutiennent que les concepteurs de systèmes travaillent à court, très court terme. Ils veulent maximiser les profits à brève échéance. Les répondants ajoutent que les propriétaires d'entreprises et leurs gestionnaires voient eux aussi souvent à très court terme. Un permanent donne l'exemple de l'industrie des pâtes et papier où on s'occupait de profit à court terme sans se soucier qu'à moyen et long termes les moyens de production devenaient désuets et l'industrie non compétitive. Un autre permanent souligne aussi que même la technologie pousse les concepteurs à travailler à court terme, puisque l'évolution se fait en termes de mois; il en résulte que, à court terme, ces mêmes systèmes deviennent souvent désuets et à reprendre. 
En ce qui touche l'implantation, on souligne quelquefois qu'en milieu gouvernemental on identifie le personnel touché, sans toutefois que cela se répercute vraiment dans des plans d'adaptation particuliers; tous les permanents rencontrés disent que non seulement on ne détermine pas le personnel qui sera touché par les systèmes, mais que ce n'est même pas une préoccupation des concepteurs.

Les réponses relatives au temps consacré par les concepteurs à l'implantation oscillent de peu à pas du tout, soulignant dans ce dernier cas que l'initiative est entièrement laissée à la discrétion des gestionnaires. Donc, contrairement à la théorie en systèmes d'information qui souligne l'importance de l'implantation, il ne semble pas dans la pratique qu'on vive une situation dans ce sens.

\section{CONCLUSION}

Nous avons voulu, dans cette étude, exposer et discuter une stratégie d'étude exploratoire et l'illustrer par la suite à l'aide d'un cas visant à confirmer ou infirmer l'intérêt de poursuivre plus avant l'étude de l'influence du groupe des usagers-opérateurs dans le succès de l'implantation des systèmes d'information organisationnels. Une étude exploratoire de cette question nous était apparue a priori justifiée par le fait que les enjeux monétaires et sociaux qui la sous-tendent étaient de taille et la littérature en systèmes d'information relativement muette à son sujet. Nous avons proposé une stratégie exploratoire qui consiste à dresser une esquisse préliminaire du système d'action concret dans lequel sont impliqués les principaux groupes d'acteurs, parties prenantes à la problématique, pour procéder par la suite à un début de validation de cette esquisse. L'usage de cette stratégie dans le présent cas a nettement confirmé l'intérêt de poursuivre plus avant les recherches. Il ressort clairement en effet que par l'entremise de leur syndicat local et de leur affiliation à des centrales syndicales, les usagers-opérateurs contrôlent des zones d'incertitude telles qu'ils peuvent, s'ils le désirent et se mobilisent, remettre en cause le succès du processus d'implantation de systèmes. La poursuite de cette étude, qui implique un processus de validation similaire auprès des autres groupes d'acteurs identifiés dans l'esquisse préliminaire du système d'action concret, s'impose donc afin que le rôle de chacun puisse apparaître dans une perspective plus juste, plus équilibrée et que des conclusions plus définitives puissent être tirées. C'est d'ailleurs dans ce sens que nous prévoyions maintenant diriger nos efforts de recherche. Mais cela ne relève plus alors du cadre strict d'une étude exploratoire.

À notre avis, un des intérêts de construire d'abord une esquisse préliminaire du système d'action concret et, par la suite, de tenter de procéder à 
une validation par étapes réside précisément dans le fait que cette stratégie, d'abord préliminaire, peut être interrompue s'il s'avère que la question posée est peu intéressante. Dans le cas contraire, elle peut être poursuivie et devenir alors un véritable instrument de formulation et de résolution de problèmes. En effet, pour Crozier et Friedberg (1977), il ne peut y avoir de véritable changement sans modifier profondément les règles du jeu de sorte que le système d'action concret $s$ 'en trouve significativement transformé. Un changement en profondeur comporte des risques pour toutes les parties, car il constitue une remise en question des règles du jeu, des sources de pouvoir et des marges de liberté d'action qu'il engendrerait pour les remplacer par d'autres qui peuvent s'avérer plus intéressantes certes, mais plus incertaines. Il fait disparaître certaines zones d'incertitude pour les remplacer par d'autres et change la source du contrôle sur ces zones. Mais malgré ces risques, nous partageons la philosophie de ceux qui pensent que les individus ne sont pas, par définition, réfractaires à tout changement. Comme le soulignent justement Crozier et Friedberg (p. 334): «Les membres d'une organisation ne sont pas en effet, attachés de façon passive et bornée à leurs routines. Ils sont tout à fait prêts à changer très rapidement s'ils sont capables de trouver leur intérêt dans les jeux qu'on leur propose».

Le changement nécessité par la résolution du problème qui a été examiné ici passe par la nécessaire prise de conscience explicite par les divers groupes d'acteurs, de la nature du système d'action concret dans lequel ils sont impliqués et la reconnaissance par eux du fait que ce jeu est à somme négative, c'est-à-dire que tous y sortent perdants. La reconnaissance de cet état de chose, sans être la garantie d'un changement réussi, est tout de même une condition nécessaire. En d'autres mots, on ne peut espérer aucun changement d'une partie à un système d'action concret à moins que cette partie ne réalise d'abord que le jeu auquel elle participe est à son désavantage. Lorsque toutes les principales parties prenantes le réalisent, nous avons là réunies les meilleures conditions pour qu'une recherche de solution soit perçue comme légitime et appropriée.

La poursuite du processus de validation par étapes avec les principaux groupes d'acteurs identifiés dans l'esquisse préliminaire du système d'action concret que nous avons présentée s'avère, selon nous, un excellent moyen de forcer cette prise de conscience collective. En ce sens, la stratégie d'étude exploratoire proposée ici se transforme peu à peu en instrument de résolution de problème et évite ainsi la difficulté liée au problème de discontinuité mentionné au début de l'article. 


\section{RÉFÉRENCES}

ALLAIRE, Luc, «Face au changement», Mouvements, été 1985, pp. 37-42.

ARGYRIS, C., «Management Information Systems: The Challenge to Rationality and Emotionality», Management Science, vol. 17, no 6, février 1971, pp. B-275-B-292.

ATTEWELL, Paul, James RULE, «Computing and Organizations: What we Know and What we Don't Know, Communications of the ACM, vol. 27, no 12, décembre 1984, pp. 1184-1192.

BERARDO, Donald J., "Are DP Professionals More Likely to Divorce?», Data Management, vol. 21, no 9, septembre 1983, pp. 16-19.

BETCHERMAN, Gordon, Kathryn MCMULLEN, La technologie en milieu de travail, Centre d'édition du Gouvernement du Canada, Ottawa, 1986, 45 pp.

BOSTROM, Robert P., J. Stephen HEINEN, «MIS Problems and Failures: A Socio-Technical Perspective: The Causes», MIS Quarterly, septembre 1977, pp. 17-32.

BUREAU INTERNATIONAL DU TRAVAIL, Les partenaires sociaux face au changement technologique, 1982-1985, Organisation internationale du travail, Genève, $1986,420 \mathrm{pp}$.

CARUTH, Don et al., «Overcoming Resistance to Change», Advanced Management Journal, vol. 50, no 3, été 1985, pp. 23-27.

CHAUMEL, Jean-Louis, Recueil de communications sur l'implantation des changements technologiques, Université du Québec à Rimouski, Rimouski, 1984, 25 pp.

CIBORRA, C., P. MAGGIONILI, "User-oriented Education in Informatics: The Experiences of some European Trade Unions», Computers in Education, 1981, pp. 497-502.

CLEGG, Chris, Nigel KEMP, «Information Technology: Personnel, Where Are You?», Personnel Review, vol. 15, no 1, 1986, pp. 8-15.

CLICHE, Vincent, «L'informatique dans l'appareil gouvernemental québécois», Le Soleil, samedi, 20 juin 1987, p. B-3.

CROZIER, Michel, Erhard FRIEDBERG, L'acteur et le système, Éditions du Seuil, Paris 1977, $437 \mathrm{pp}$.

DESANCTIS, Gerardine, James F. COURTNEY, «Toward Friendly User MIS Implementation", Communications of the ACM, vol. 26, no 10, octobre 1983, pp. 732-738.

DOMMERGUES, Pierre, «Les syndicats français et américains face aux mutations technologiques», Le Monde diplomatique, janvier 1984, pp. 2-3.

DUSSAULT, Gaston, "L'implantation des nouvelles technologies ne fait pas oublier la rentabilité», Le Journal du Travail, septième année, no 2, mars 1985, pp. 8-9. 
FERRAND, Dominique, Maurice LANDRY, «Pratiques du développement du système d'information comme problème non-structuré», Actes des Journées nationales des I.A.E.: Futur et gestion de l'Entreprise, Poitiers, novembre 1987.

FILIATRAULT, P., La réalisation du changement planifié (cours préparé pour la Commission de la fonction publique au Canada), Faculté des Sciences de l'Administration de l'Université Laval, $28 \mathrm{pp}$.

FRANZ, Charles R. et al., «User Response to an Online Information System: A Field Experiment», MIS Quarterly, vol. 10, no 1, mars 1986, pp. 29-42.

FRECHET, Guy et al., L'informatique et ses impacts socio-professionnels: le cas de la fonction publique québécoise, Université Laval, Québec, 1987, 4 volumes, 872 pp.

GAGNON, Mona-Josée, «Les changements technologiques: pour ne pas arriver trop tard», Vie Ouvrière, vol. 34 , no 187 , août 1985, pp. 28-29.

HIRSCHHEIM, R.A., Office Automation: A Social and Organizational Perspective, Wiley, Chichester, 1985, 327 pp.

KEEN(a), Peter G., «Managing Organizational Change: The Role of MIS», Proc. 6th and 7th Annual Conference of the Society for Management Information Systems, J.D. White ed., University of Michigan, Juillet 1976, pp. 129-134.

(b), «Information Systems and Organizational Change», Communications of the $A C M$, vol. 24, no 1, janvier 1981, pp. 24-33.

- _ _ - «Communications in the 21st Century: Telecommunications and Business Policy», Organizational Dynamics, Automne 1981, pp. 54-67.

_____, «Computers and Managerial Choice», Organizational Dynamics, Autumn 1985, pp. 35-49.

KEEN, P.G.W., M. SCOTT MORTON, «Implementation: the Management of Organizational Change», Decision Support Systems, Addison-Wesley, 1980, pp. 189-211.

KLING, R., «Social Analyses of Computing: Theoretical Perspectives in Recent Empirical Research», Computing Survey, mai 1980, pp. 61-110.

LAFARGUE, Yves, «La panne ou la négociation», Projet, no 197, mars 1986, pp. $11-18$.

LAMOUREUX, Daniel, L'introduction de technologies nouvelles dans l'entreprise: facteurs et conséquences, Institut national de productivité, Montréal, 1983, $53 \mathrm{pp}$.

LUCAS, Henry Jr., Why Information Systems Fail, Columbia University Press, New York, 1975, 130 pp.

LYONS, Michael, «The DP Psyche», Datamation, août 1985, pp. 103-110.

MCKENNEY, James L., Peter G.W. KEEN, «How Managers’ Minds Work», Harvard Business Review, vol. 52, no 3, mai-juin 1974, pp. 79-90.

MERCIER, Jean, «L'informatique, ses 'filtres' et ses effets», Relations industrielles, vol. 41, no 2, 1986, pp. 299-315. 
MILES, Mary, «Dealing with Resistance to Change», Computer Decisions, vol. 15, no 5, mai 1983, pp. 202-206.

MINISTĖRE D'ÉTAT SCIENCES ET TECHNOLOGIE, La Conférence le Canada demain, Centre d'édition du gouvernement du Canada, Ottawa, 1984, 89 pp.

MORIN, Gilles, «On peut atténuer le choc post-implantation informatique», Le Devoir, mardi, 11 février 1986, p. 10.

MUMFORD, Enid, Frank LAND, John HAWGOOD, A Participative Approach to Forward Planning and System Change, Percy House, England, 1978, 28 pp.

NEW, J. Randolph, Daniel D. SINGER, «Understanding Why People Reject New Ideas Helps IEs Convert Resistance Into Acceptance», Industrial Engineering, vol. 15 , no 5, mai 1983, pp. 51-57.

OLSON, Margrethe H., «New Information Technology and Organizational Culture», MIS Quarterly, édition spéciale 1982, pp. 71-92.

OLSON, Margrethe H., Jon A. TURNER, «Rethinking Office Automation», Proceedings, Conference on Information Systems, Indianapolis, 1985, pp. 259-269.

PAYEUR, Christian, «L'organisation du travail: l'enjeu d'une sortie de crise», Mouvements, automne 1983, pp. 56-57.

-_- Nouvelles technologies et conditions de travail: matériaux pour une stratégie syndicale, Communications CEQ, mars 1985, 66 pp.

POPPEL, Harvey L., «Qui a besoin du bureau de demain?», Harvard-l'expansion, no 29, été 1983, pp. 6-17.

ROBEY, Daniel, Dana FARROW, «User Involvement in Information Systems Development: A Conflict Model and Empirical Test», Management Science, vol. 28, no 1 , janvier 1982, pp. 73-85.

ROPP, Kirkland, «Technology and Employment», Personnel Administrator, vol. 32, no 2, février 1987, pp. 81-84.

ROY, Marie Christine, Système d'information, locus de contrôle et cadres intermédiaires de la région de Québec, document présenté au congrès de l'ACFAS, 1986.

SALOMON, Jean-Jacques, Prométhée empêtré, la résistance au changement technique, Pergamon Press, Paris 1982, 174 pp.

SAMSON, Michel, "Conférence sur l'électronique et l'informatique: Les participants sont restés sur leur faim», Le Soleil, samedi, le 13 avril 1985, pp. A-6.

SANDBERG, Ake, «Socio-Technical Design, Trade Union Strategies and Action Research», dans Research Methods in Information Systems, E. Mumford et al. (éditeurs), North-Holland, 1985, pp. 79-99.

TREMBLAY, Gaétan et al. (Institut candien d'éducation des adultes), «Négocier le virage technologique», Magazine $C E Q$, vol. 2, no 3, printemps 1983, pp. 15-22.

VALLÉE, Guylaine, Les changements technologiques et le travail au Québec: un état de situation, Commission consultative sur le travail et la révision du Code du travail, les publications du Québec, 1986, $178 \mathrm{pp}$. 
WHITE, Kathy Brittain, «MIS Project Teams: An Investigation of Cognitive Style Implications», MIS Quarterly, juin 1984, pp. 95-101.

ZMUD, Robert W., «An Examination of 'Push-Pull' Theory Applied to Process Innovation in Knowledge Work», Management Science, vol. 30, no 6, June 1984, pp. 727-738.

ZMUD(a), Robert W., James F. COX, «The Implementation process: A Change Approach», MIS Quarterly, June 1979, pp. 35-43.

ZMUD(b), Robert W., "Individual Differences and MIS Success: A Review of the Empirical Literature», Management Science, vol. 25, no 10, octobre 1979, pp. 966-979.

\section{Technological Change A Strategy for an Exploratory Study}

Two distinct categories can be established in the field of exploratory studies. First, there are studies on subjects that are relevant but which, for various reasons, have been left more or less unexplored. The second category of exploratory studies comes above the first category since these studies revolve around issues which relevance has not been yet supported in a way that would justify thorough consideration and adequate investment, even if a certain form of argumentation could be elaborated on their potential interest. To work with the second category of exploratory studies, it is necessary to create an approach that will confirm or invalidate their potential interest and, in the case of a confirmation, determine the orientation of the subsequent studies.

A problem may arise from the use of the last mentioned category of strategies. When these strategies do confirm the potential interest of the studies, they have to be given up for other strategies that are best suited to carry out the research. Consequently, new resources as well as additional time are required. In both scientifically and professionally oriented fields, this problem could have a negative impact since the researches carried out in these fields often give rise to more immediate concerns and expectations. In our opinion, the problem arising from this discontinuity is not necessarily unavoidable. 
In this article, our objective is to set out and examine an investigation strategy that we already used to tackle a problem falling in the second category of exploratory studies and relating to the implementation of technological changes in unionized environments. This strategy uses concepts such as «concrete action system», «power», and «uncertainty zone» which form the basis of the theory of organizations elaborated by Crozier and Friedberg (1977). This approach avoids the discontinuity problem previously mentioned and indicates interesting investigation paths, when such paths exist. Moreover, it does not require a large investment at the beginning of the research. Finally, it seems to us that this strategy can easily be used and adapted for several research problems that arise in the field of relations in the workplace.

\section{INDUSTRIAL RELATIONS JOURNAL}

\section{Edited by Brian Towers}

The Industrial Relations Journal, established in 1970, is a leading UK-based journal with an international flavour, which aims to monitor, report and predict significant developments in industrial relations for practitioners, policy-makers and academics. Articles in the Industrial Relations Journal have included studies of trade unions in modern industrial society, the industrial relations practices of foreign-owned multinationals, and the decline of mass production and union survival in the USA. The journal has recently launched a new feature on Trends and Developments in Industrial Relations and the Law. The annual subscription includes four issues of $I R J$ plus two companion issues of New Technology, Work and Employment, edited by Colin Gill.

\section{Recent articles include:}

Trends and developments in industrial relations law: The Employment Act 1988 KARL MACKIE

\section{Japanisation: a lack of chemical reaction} MICK MARCHINGTON

Working-time reduction: a survey of the Australian experience JOHN MANGAN and JOHN STEINKE

Industrial Relations Journal is published in Spring, Summer, Autumn and Winter plus free subscription to New Technology, Work \& Employment (published Spring and Autumn)

Subscription Rates Volume 20, 1989

Individuals: $£ 50.00$ (UK) $£ 59.50$ (overseas) US\$99.00 (N. America)

Institutions: 265.50 (UK) $£ 78.50$ (overseas) US $\$ 129.50$ (N. America)

\section{Basil Blackwell · Journals Marketing Manager,} 108 Cowley Road, Oxford OX4 1 JF UK or, Journals Marketing Manager, 432 Park Avenue South, NY 10016, USA. 